\title{
Implementing recommendations of the Columbia Accident Investigation Board - development of on-orbit IR thermography
}

\author{
Brian P. Ottens ${ }^{* a}$, Bradford Parker ${ }^{a}$, Ryan Stephan ${ }^{b}$ \\ NASA Goddard Space Flight Center, Greenbelt, MD 20771 \\ bASA Langley Research Center, Hampton Roads, VA 23681
}

\begin{abstract}
One of NASA's Space Shuttle Return-to-Flight (RTF) efforts has been to develop thermography for the on-orbit inspection of the Reinforced Carbon Carbon (RCC) portion of the Orbiter Wing Leading Edge (WLE). This paper addresses the capability of thermography to detect cracks in RCC by using in-plane thermal gradients that naturally occur on-orbit. Crack damage, which can result from launch debris impact, is a detection challenge for other on-orbit sensors under consideration for RTF, such as the Intensified Television Camera and Laser Dynamic Range Imager. We studied various cracks in RCC, both natural and simulated, along with material characteristics, such as emissivity uniformity, in steady-state thermography. Severity of crack, such as those likely and unlikely to cause burn through were tested, both in-air and in-vacuum, and the goal of this procedure was to assure crew and vehicle safety during reentry by identification and quantification of a damage condition while on-orbit. Expected thermal conditions are presented in typical shuttle orbits, and the expected damage signatures for each scenario are presented. Finally, through statistical signal detection, our results show that even at very low in-plane thermal gradients, we are able to detect damage at or below the threshold for fatality in the most critical sections of the WLE, with a confidence exceeding 1 in 10,000 probability of false negative.
\end{abstract}

Keywords: Space Shuttle Return to Flight Thermography

\section{INTRODUCTION}

The breakup of the Space Shuttle Columbia in 2003 resulted in the loss of seven human lives, anorbiter, significant time without STS flights, and a reconfiguring of many NASA operations. An independent board which formally became known as the Columbia Accident Investigation Board (CAIB), was assembled to investigate the disaster. The CAIB addressed both the physical and organizational causes of the accident. .

The CAIB concluded that the cause of the accident was a breach of the Thermal Protection System (TPS), occurring near panel 8 of Reinforced Carbon-Carbon (RCC), on the leading edge of the left wing. ${ }^{1}$ As a result of their investigation, the CAIB made a series of recommendations to NASA that would reduce or eliminate the likelihood of a similar disaster in the future ${ }^{1}$. Recommendations were numerous and far reaching, and NASA administrator Sean O'Keefe has committed to implementing them to the best of NASA's ability before the next STS flight ${ }^{2}$.

One of the CAIB recommendations specifically dictates an on-orbit inspection of the TPS. Recommendation, \#R6.4-1 reads:

For missions to the [ISS], develop a practicable capability to inspect and effect emergency repairs to the widest possible range of damage to the [TPS], including both tile and [RCC], taking advantage of additional capabilities available when near to or docked at the ISS.

For non-Station missions, develop a comprehensive autonomous (independent of Station) inspection and repair capability to cover the widest possible range of damage scenarios.

Accomplish an on-orbit [TPS] inspection, using appropriate assets and capabilities, early in all missions.

\footnotetext{
*Brian.Ottens@nasa.gov phone (301)286-3091 fax (301)286-1672 http://www.nasa.gov
} 
The ultimate objective should be a fully autonomous capability for all missions to address the possibility that an ISS mission fails to achieve the correct orbit, fails to dock successfully, or is damaged during or after undocking. ${ }^{3}$

This paper will discuss the results of a feasibility study that addressed the use of Infrared (IR) thermography to perform on-orbit inspection of the TPS RCC. This study was performed in direct response to the above CAIB recommendation.

\subsection{Background}

The initial response to the $\mathrm{CAIB}$ recommendation for on-orbit inspection was the development of a flight inspection system, the Orbiter Boom Sensor System (OBSS). The OBSS (Figure 1)consists of a boom extension to the current Shuttle Remote Manipulator System (SRMS), the Integrated Sensor Inspection System (ISIS), and various infrastructure modifications (both on the orbiter and the ground) to support each.

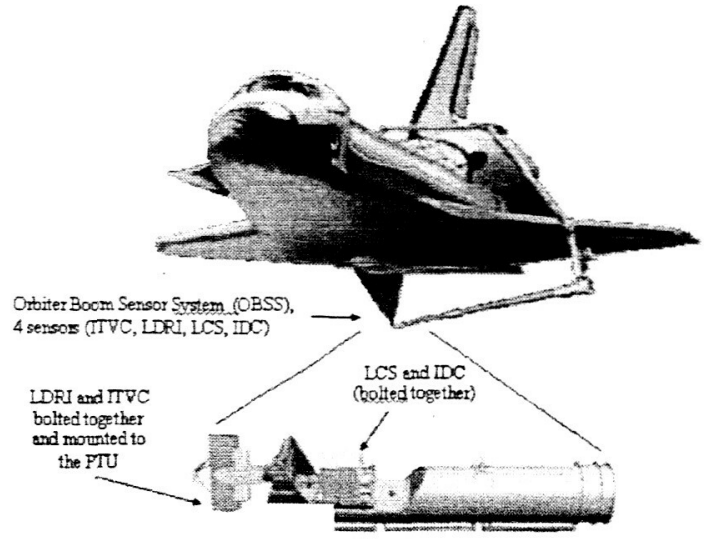

Figure 1: The OBSS

As one can see in Figure 1, the boom and SRMS position ISIS and move it about so that the TPS is within its field of view. Exact flight configurations and schedules will not be discussed in this paper, however, ISIS generally consists of the Laser Dynamic Range Indicator (LDRI), which is affixed to the Intensified Television Camera (ITVC). Both of those are mounted to the Pan-and-Tilt Unit (PTU) for 2-DOF motion independent of the boom, and a fixed-to-boom Laser Camera Head $(\mathrm{LCH})+$ periphery device. The periphery device interface was designed so that any dimensionally, thermally, and electrically suitable instrument could be affixed to the $\mathrm{LCH}$, but the only periphery device currently under development is the IDC, a 2 Mpix digital camera with interchangeable lenses

An advantage of using the OBSS is that many instruments have flight heitage, so performance characteristics can be reasonably estimated and development risk is low. This suite of instruments was a good match to the baseline TPS inspection requirements. Those inspection requirements for the RCC portion of the TPS were, depending on location, the detection $0.25-3$ inch features. ${ }^{4}$

Concurrrent with the development of the OBSS, NASA was conducting an RCC damage tolerance test program in response to a separate CAIB recommendation. As this test program progressed, it became apparent that the baseline inspection requirements may change. The testing confirmed that other types of RCC damage such as cracks, silicon carbide coating loss, and delaminations could also pose a threat. This finding accelerated NASA's investigation of onorbit inspection techniques to complement the OBSS, and based on its success in ground based testing, IR thermography showed promise. Hence, NASA brought together a group from Johnson Space Center (JSC), Langley Research Center (LaRC), and Goddard Space Flight Center (GSFC) to determine the feasibility of on-orbit IR thermography. JSC provided guidance and expertise on the orbiter and the problem; LaRC provided expertise in NDE, IR applications, and the configuration for testing; and GSFC provided expertise in NDE, IR science, and instrument development.

\subsection{Initial Assessment of Feasibility}


This task was accomplished by installing a damaged RCC panel 6 (from Discovery) into the setup shown in Figure 2 , and acquiring IR measurements at temperature gradients of $>5^{\circ} \mathrm{C} /$ in. The thermal gradients were established by warming the top portion of the RCC panel with quartz lamps while the bottom portion of the panel viewed a cold chamber shroud. The RCC damage was the result of hypersonic foam impact testing ${ }^{5}$, and because high-velocity impacts have been shown to create cracks in $\mathrm{RCC}^{6}$, it was a good sample to gauge feasibility. Furthermore, it represented damage manifesting itself in a narrow crack, which is, as previously alluded to, is a particularly difficult challenge for direct measurement techniques. One can see a detail of this damage in Figure 3.
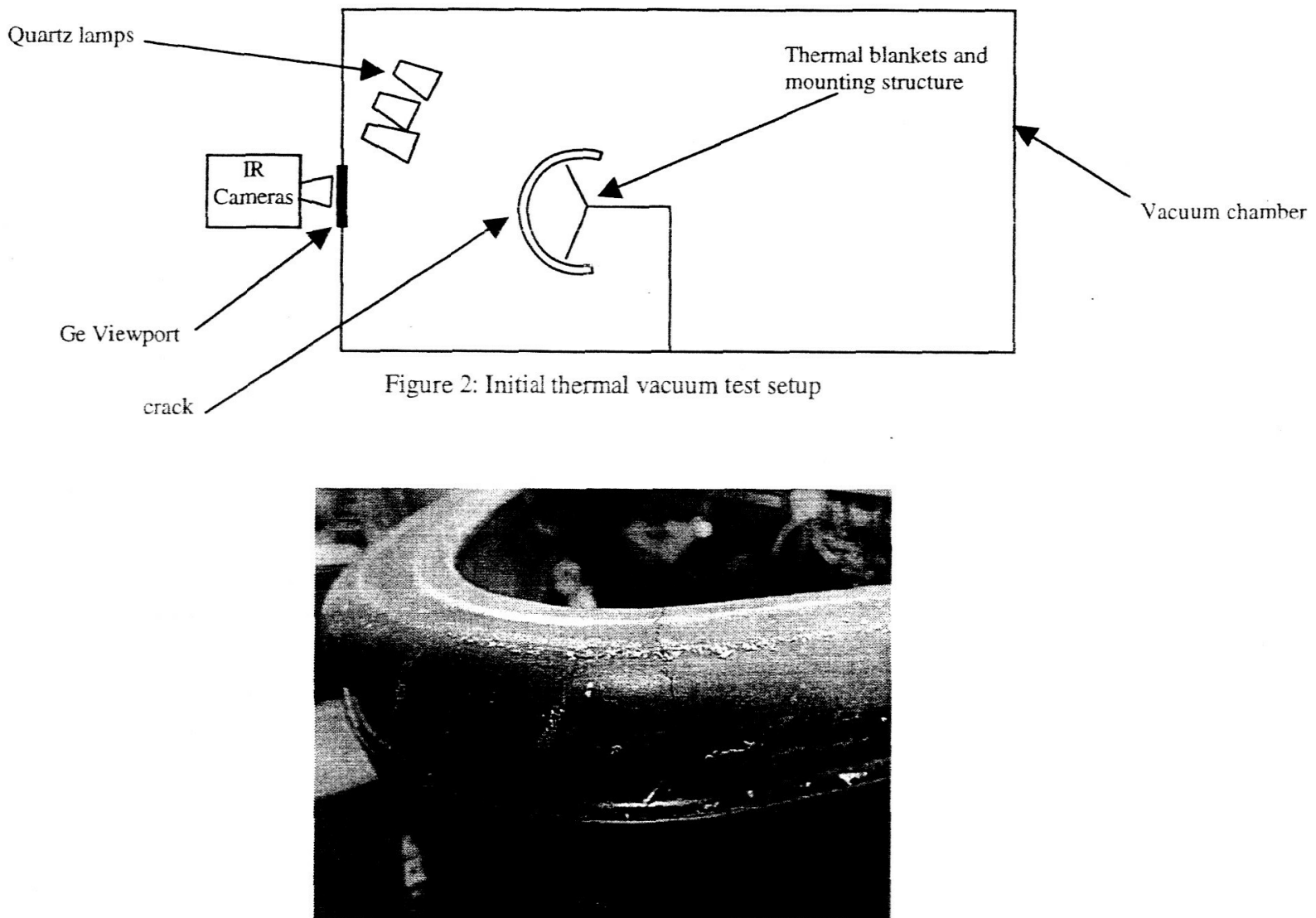

Figure 3: Close-up of damage (CAIB Photo by Rick Stiles 2003)

While participating in this test, plans for implementation (if successful) were not firm. So a selection of imagers that covercd various wavelengths and sensing technologies were employed, as it allowed significant flexibility in determining schedule for instrument delivery and full inspection requirements. The two commercial cameras in the setup were good representations of what has been used for laboratory based NDE, and the two prototype cameras were representations of what the GSFC IR group could turn around on short notice and/or with significant flight heritage. This allowed the team to consider a variety of options representing little development risk and past measurement experience (see appendix A for camera specifications).

The two commercial cameras produced the best images of the crack damage in the RCC panel, and an example thermal image is shown in Figure 4. The crack created an impediment to in-plane heat flow, and as a consequence, created a perturbation in the panel top-to-bottom thermal profile that can be visually detected in the thermal image. 


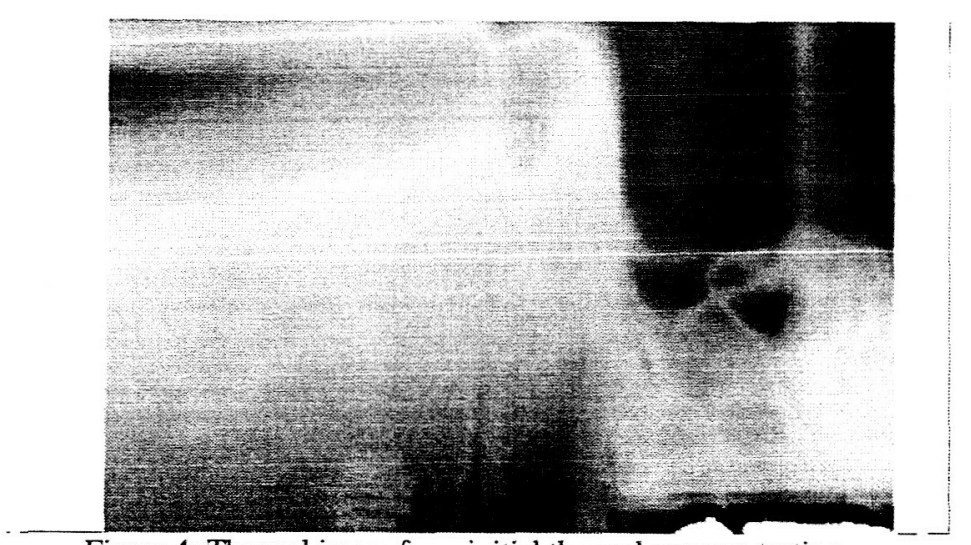

Figure 4: Thermal image from initial thermal vacuum testing

\section{CONCEPT MATURATION}

As a result of these promising test results, the groups at GSFC and LaRC were tasked to further develop the IR inspection concept. The emphasis of this work was to study crack detection, as this was a perceived weakness of the OBSS, and the CAIB impact tests had shown that cracking was a realistic possibility. One parameter of study was the on-orbit thermal environment, as it was necessary to establish reasonable expectations of orbital magnitude and direction of thermal gradient. The groups were also tasked to study the effects of emissivity variations, off angle imaging (the RCC panels have extreme curvature), and camera spatial resolution.

In order to study narrow cracks, we obtained a 6"x6" sample of RCC from the precious supply available. We then cut four slots into this sample using a 0.006 inch wide, diamond cutting wheel. The four slots, which were arranged in a pinwheel fashion, had depths of $92,66,42$ and 20 percent of the sample's thickness. The profile of the slots corresponded to the profile of the portion of the 3 inch diameter cutoff wheel that plunged into the sample (Figure 5).

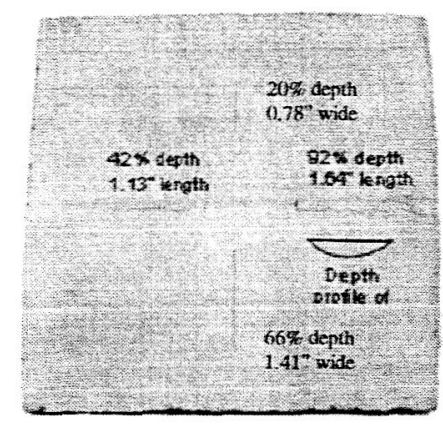

Figure 5: Simulated damage profiles

We believe this sample differed compositionally from the RCC used for flight only by a lack of TEOS impregnation and Type-A sealant on the outermost surface (Figure 6). Therefore, the emittance of the test coupon was slightly higher and the thermal conductivity slight lower than flight RCC.

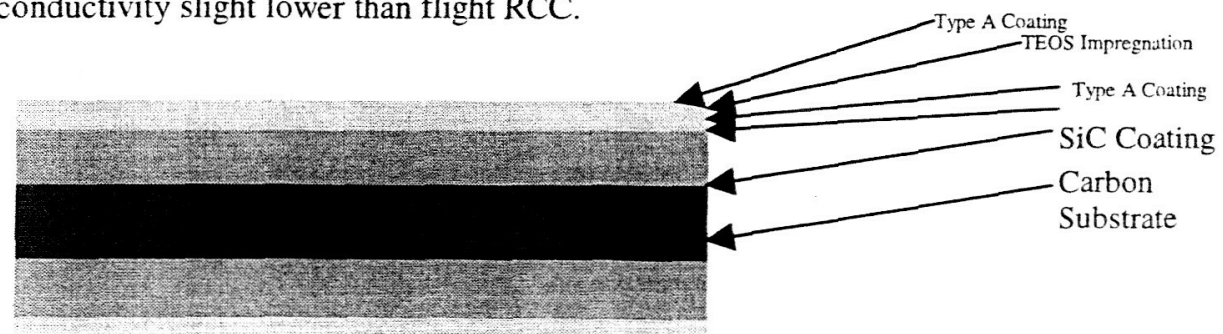

Figure 6: RCC 
For the in-air phase of our measurements, we induced thermal gradients of various magnitudes across the face of this sample as shown in Figure 7. For all of these measurements, sufficient time was allocated to allow $\Delta T e m p / \Delta t i m e$ to be very small, and gradients were measured using the IR camera. Thermal images were then captured with the Indigo Merlin camera described in appendix A, and those images were analyzed using several methods.

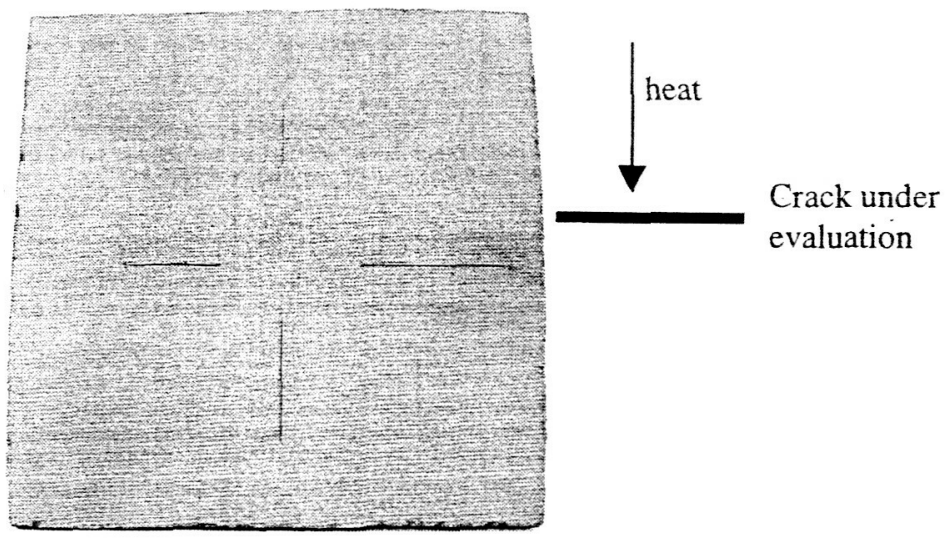

Figure 7: Direction of thermal gradients

The first goal of these measurements was to determine the magnitude of thermal gradient required to detect the simulated cracks of various depths. Our second goal was to refine the setup and analysis procedure, thereby reducing the temperature gradient necessary to setup a proper on-orbit measurement. This would help guarantee inspectability in every section of RCC, and accomplish the survey as quickly as possible.

Our first tests were performed using rather high gradients (6-12 degrees $C$ per inch), and results were analyzed.- 3-D temperature plots of the sample were generated using MATLAB's surf command (Figure 8), and as expected, abnormalities in temperature profile were apparent in the general area of the slots. Then, a colleague recommended we compute a simplified derivative image by ignoring $\Delta \mathrm{T} / \Delta \mathrm{x}$ in the direction perpendicular to our induced gradient (it would be very small) and simplifying the $\Delta \mathrm{T} / \Delta \mathrm{y}$ into a differential between its neighbor in the direction of temperature increase. After comparing data for a similar thermal gradient (Figures 9 and 10), the results were extremely satisfying. It shows our technique was successful in correlating a relationship between depth of crack and magnitude of surface gradient across the crack, a significant milestone in the effort.

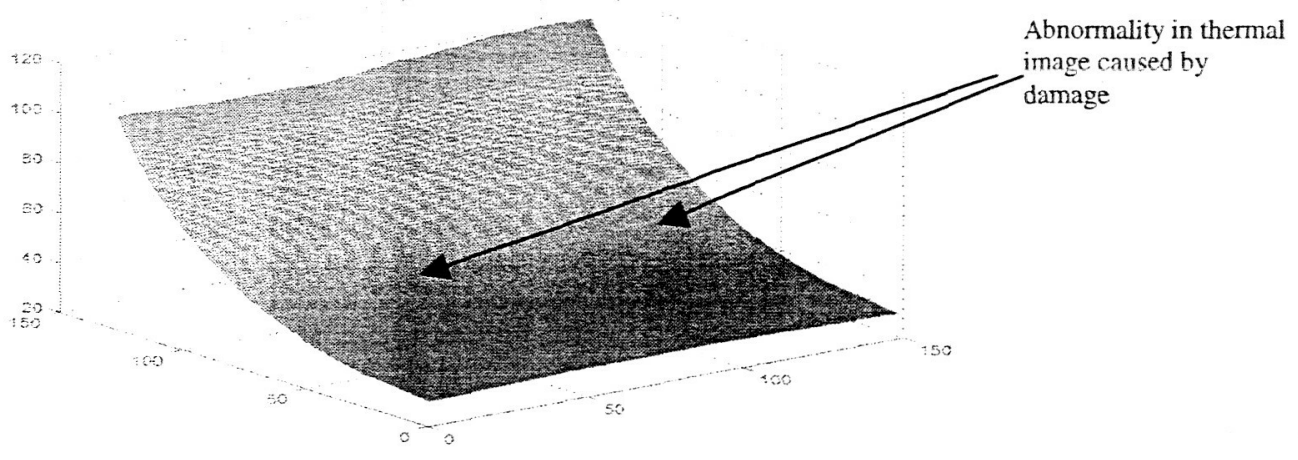

Figure 8: Temp profile using MATLAB surf command 


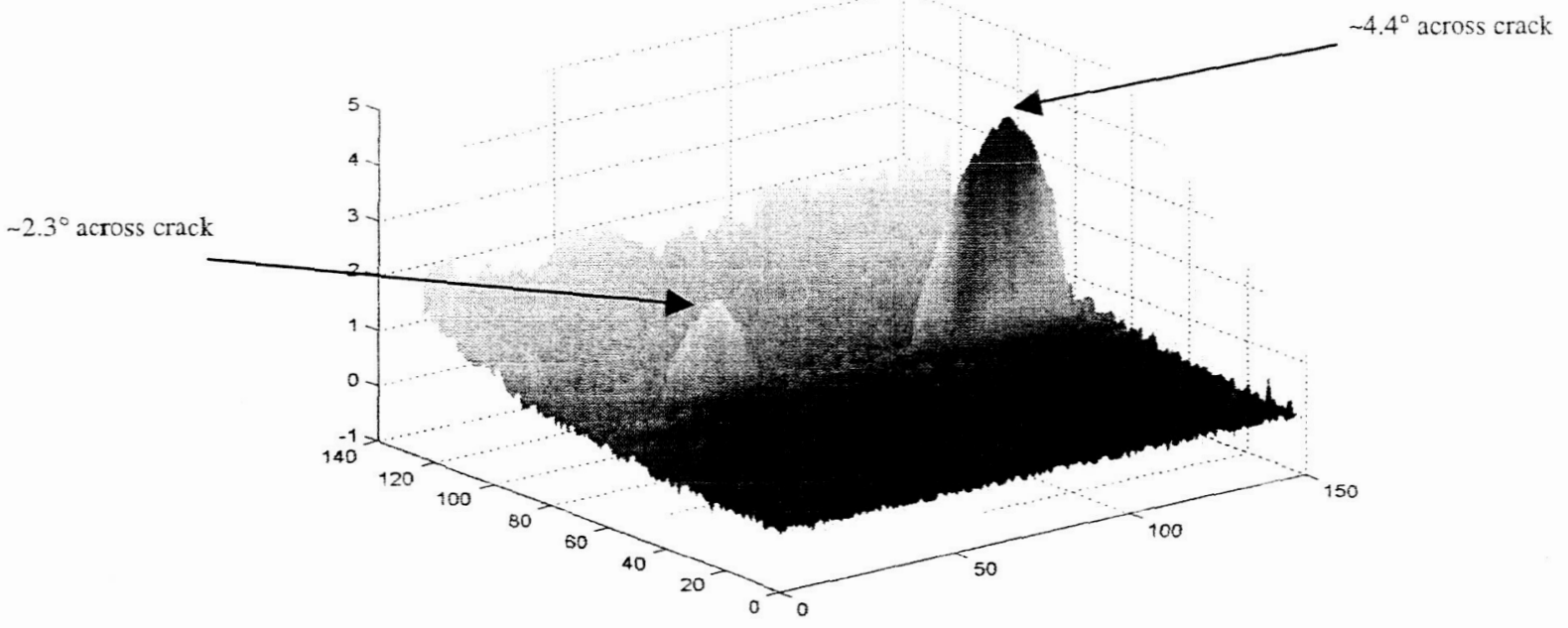

Figure 9: Plot of modified derivative filter for depth $=42 \%$ (left) and $92 \%$ (right)

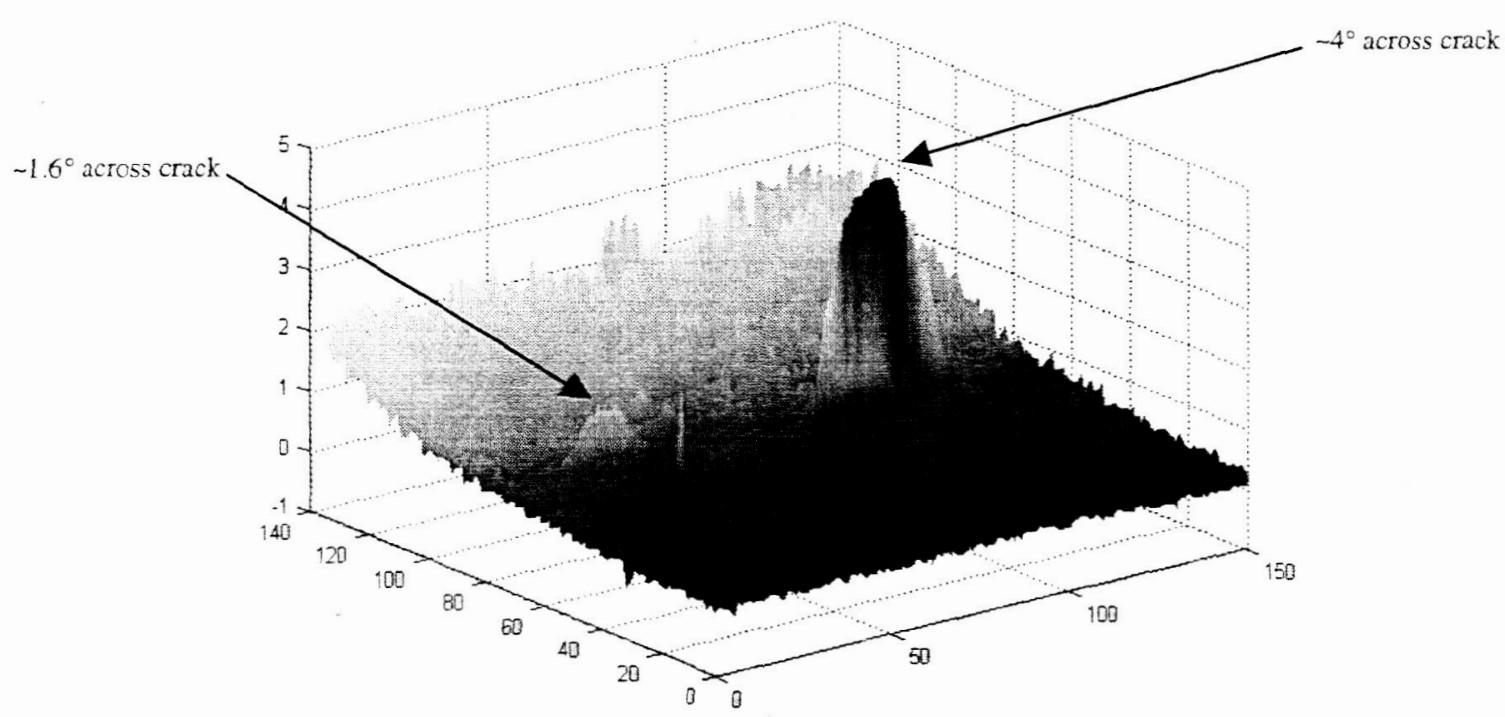

Figure 10: Plot of modified derivative filter for depth $=20 \%$ (left) and $66 \%$ (right)

Our next sequence of tests were executed with the goal of lowering the magnitude of thermal gradient necessary to detect cracks. This is important because previous gradients used in testing were somewhat high, and not likely to occur for most orbits or wing leading edge locations. The challenge was one of SNR maximization, and it was approached by studying filter coefficients, spatial resolution on the target, and minimum severity of damage necessary for confident detection. So empirical analyses were performed on the first two parameters and their effect on SNR was studied. The final parameter was set aside to allow RCC damage testing to progress.

For filter coefficients, optimization was achieved by incorporating as many sampled pixels into the filtered window as possible. Time-based averaging was generally avoided because the difficulty of keeping a camera steady was thought to be particularly challenging given the long runs and elasticity of the SRMS and boom. So any ringing or drift of the boom's position would make acquisition of an offsett-free image sequence challenging. But space-based windowing was not susceptible to the same factors, and it was indeed successful in reducing the effect of random variables while maximizing the preservation of signal. 
Our colleagues at LaRC suggested the coefficients in Figure 11, and this works well when detection is very difficult, however, at the time of this writing, the authors now believe the optimum filter to be a 2-D extension of the localized thermal impedance phenomena shown in Figure 12.

$\begin{array}{lll}0.5 & 0.7071 & 0.5 \\ 0 & 0 & 0 \\ -0.5 & -0.7071 & -0.5\end{array}$

Figure 11: Filter Coefficients Employed for Detection when Signal was not $\gg$ Noise

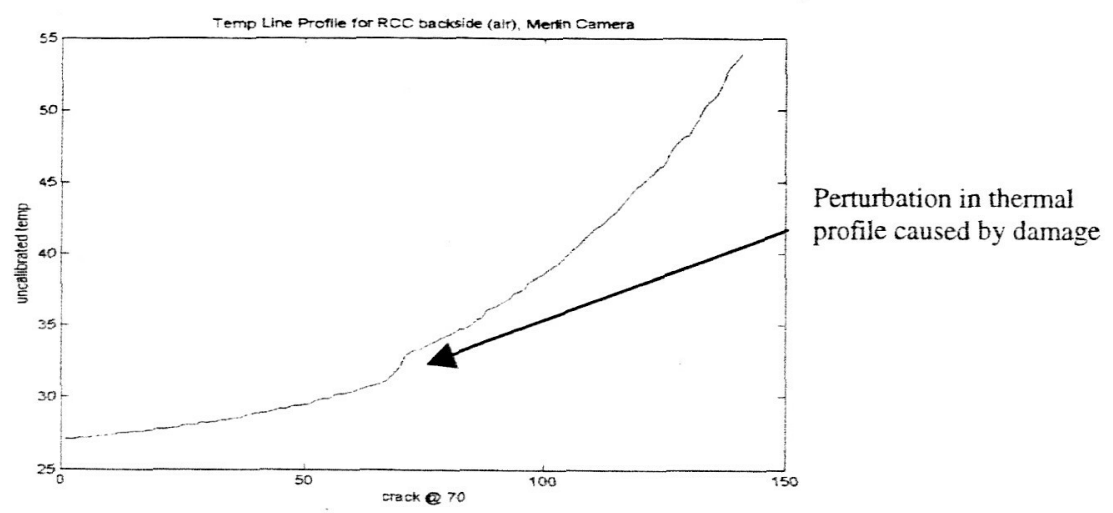

Figure 12: Temperature perturbation resulting from RCC damage

For spatial resolution, we studied its effect on the measured temperature drop across the simulated cracks. The temperature drop was obtained by the modified derivative filter previously described, and these tests showed that higher spatial resolution did improve the signal to noise ratio. In addition, we found that the reference pixel needed to be optimized for the specific spatial resolution realized during an inspection. Specifically, instead of referencing at it's nearest neighbor in the direction of the thermal gradient, we oftentimes referenced the value 2 pixels away, but still in the direction of the thermal gradient.

This analysis also contributed to our trade study in comparing microbolometer and indium antimony (InSb) sensing technologies. One of the many differences between these technologies was the size of available arrays, and one of the operational constraints initially placed on the on-orbit inspection was a 5 foot standoff distance between the OBSS and the TPS. This distance could also vary to as much as 20 feet, and we felt it was important to understand what changes a fixed focal length system would incur from changes of this magnitude. Hence, a series of tests were performed to look at the magnitude as a function of spatial resolution. The IR spatial resolution was varied by simply changing the camerato-target distance.

This data directed our development efforts by guiding our recommendation on which camera should eventually be implemented to perform the techniques we were refining. The largest micobolometers available at that time were 320 by 240 (this size later quadrupled), whereas 1024 by $1024 \mathrm{InSb}$ arrays were available. The microbolometer arrays had the advantage of being much lower in power consumption and much lower in complexity (no cryocooler), so until this time, we believed the superior choice to be microbolometer arrays. But this piece of data gave us significant confidence that the smaller arrays would be too time consuming and the imaging area would be too small to make an efficient inspection tool.

While these efforts were underway, mathematical predictions were being performed to determine exactly what gradient would be considered practical during an orbit. The first sets of predictions were performed by M. Fields of Boeing, and they provided a coarse distribution of temperatures about RCC panel \#7. However, only significant detail was distributed for centerline temperature nodes.. These models predicted that during a typical orbit, the entire centerline of the RCC panel would have in-plane thermal gradients in excess of $1 \mathrm{C} /$ inch, but were too coarse to analyze further. Note 
that this gradient was not present during the entire orbit, as during the shade portion of the orbit, gradients were almost nonexistent.

\section{VALIDATION TESTING}

After refining our procedures to utilize gradients approaching $1^{\circ} \mathrm{C} /$ in, we began to scrutinize the effect of convection. Attempts were made to quantify it, but after many unsuccessful attempts, a vacuum test was planned. Figure 13 shows physical configuration, and thermal gradients in excess of $2^{\circ} \mathrm{C} /$ in to less than 0.25 . Data was acquired on both the RCC Sample and Columbia panel \#4R, and most results showed that the elimination of convection allowed us to reduce the necessary gradient even further. It also showed led us to the conclusion that subjectivity now became the dominant factor in detection. Specifically, if we knew a crack was present, we could quickly see evidence of it, but if we did not have a prior knowledge of damage, we lost confidence that what we were seeing was actual damage and not "noise."

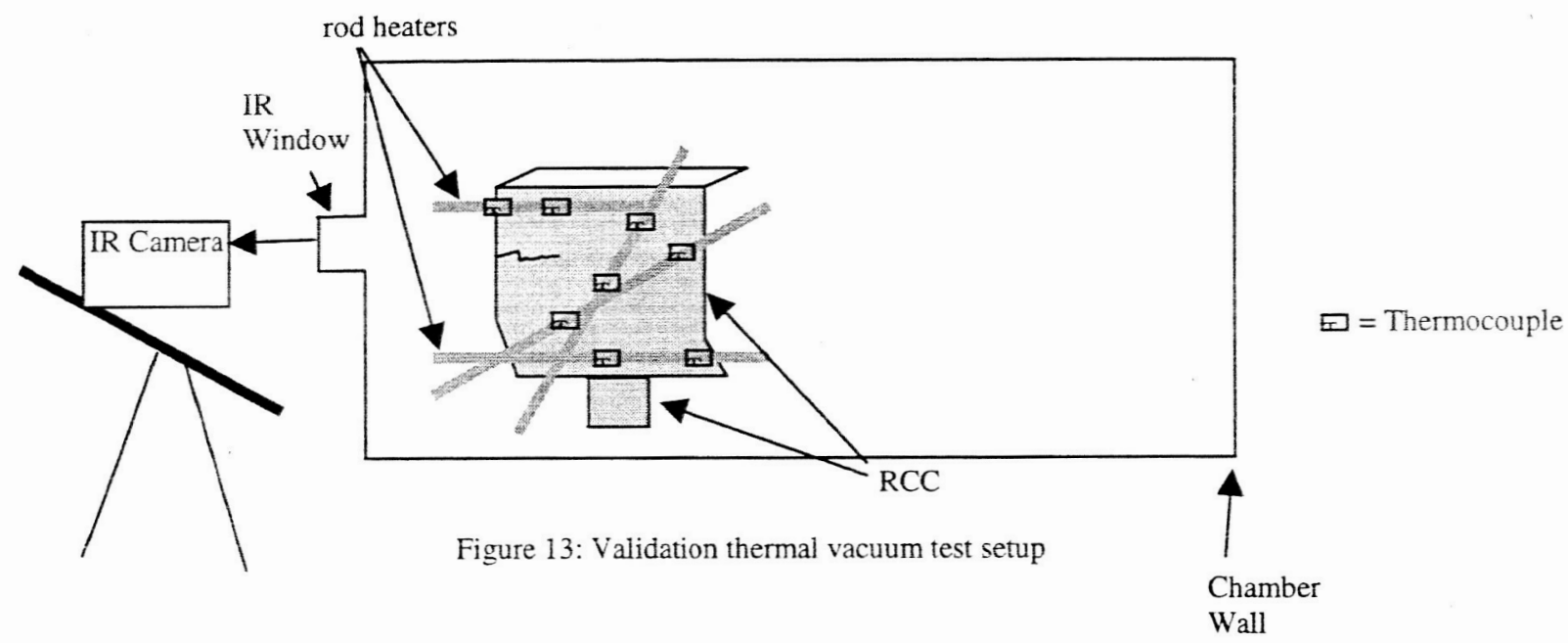

In our case, "noise" was not from random effects in the acquisition instruments, but from nonuniformities in emissivity on the sample's surface. An example of this phenomena is shown in Figure 14, and in some cases, its magnitude exceeded that of the conductive abnormality induced by the crack. This explains why one could clearly "see" a signal of damage when it was present, but to confidently conclude that it was not these nonuniformities became unreliable.

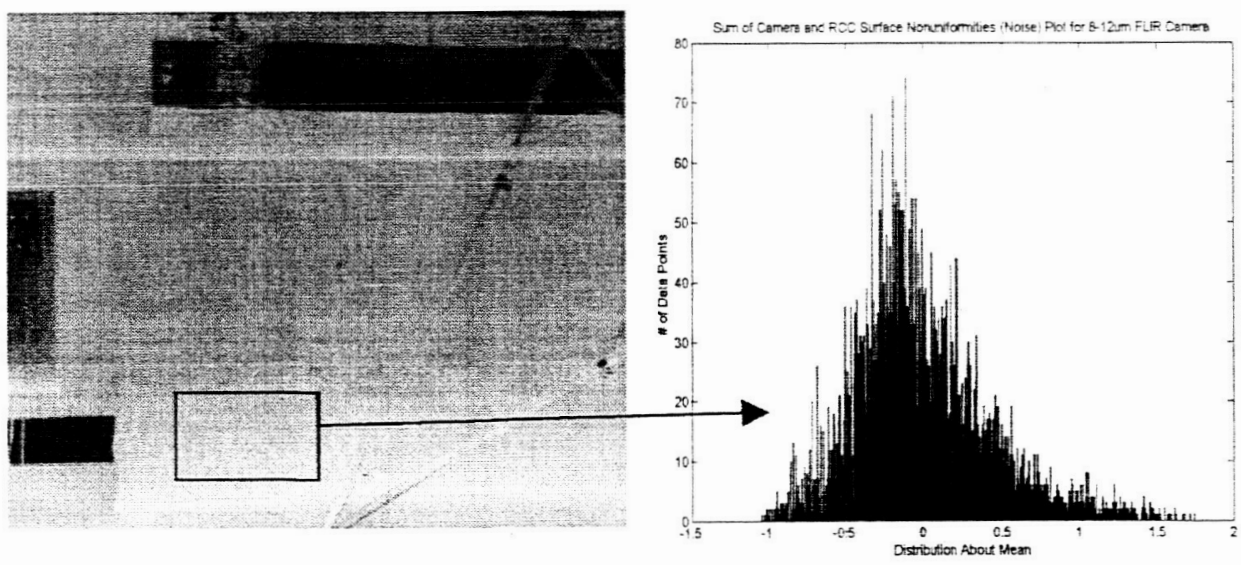

Figure 14: Example surface nonuniformities

We found that the best way to remove subjectivity from the detection process was to consider it a signal detection problem in a noisy environment, which would lay out very strict rules for detection. But to use this methodology, signal and background distributions, along with probability of error needed to be determined. Distributions were approached 
first, and luckily, nonuniformity (background) datapoints were plentiful. Brief study of them revealed that they were quite random, or at least pseudo-random, and of a distribution well approximated by a gaussian.

But characterizing the damage PDF in a similar manner was much more difficult. We only had 2 samples to analyze, and each sample only had a few dozen pixels of unnormalized damage. So any characterization of signal would be of limited scope, and we decided to make assessments without knowledge of the signal PDF. This meant that our probabilities would be for false positives only.

For this probability of error, we decided to use a value of 1 in 10,000 , which we felt was quite conservative. With this value, we performed an analysis to determine the lowest thermal gradient where a crack of minimum width and length could be detected. For the minimum dimensions, we obtained preliminary RCC damage testing results which recently became available. They suggested a need to detect 15 mil wide, 3 inch long, complete thru-cracks, so our analysis focused on the $92 \%$ depth, 1.64 inch long, 12 mil wide simulated crack in Figure 5.

After filtering the test image with the coefficients in Figure 11, we averaged the area of pixels equal to the actual length of the crack (and not solely the ones in the deepest portion of the half-penny) and compared it to a "background." The background consisted of the same length of pixels, averaged together, and offset from the damage by $4,5,6$, and 7 rows in both directions, so as to obtain a large sample of representations and avoid local effects.

What we found (Figure 15) is that even at very low gradients $\left(<0.1^{\circ} \mathrm{C} / \mathrm{cm}\right)$, there was still significant offset between the damaged locations and the background, approximating $>3 \Sigma$ from the mean. However, faith in the fidelity of our measurements was not certain past one significant figure beyond the decimal point. To verify that this gradient was within the constraints of practicality, the authors were also performing calculations to determine the suitability of $0.1^{\circ} \mathrm{C} / \mathrm{cm}$ for on-orbit inspection. Those results came out favorably, and specifically, detailed analysis of a $444 \mathrm{~km}$ altitude, beta angle of $30^{\circ}$, bay to earth roll, tail to velocity vector yaw/pitch, and orbit period of $5598.62 \mathrm{sec}$ showed that $95 \%$ of the RCC reached this gradient at some point during its cycle (Figure 16), a high enough value to validate our results.

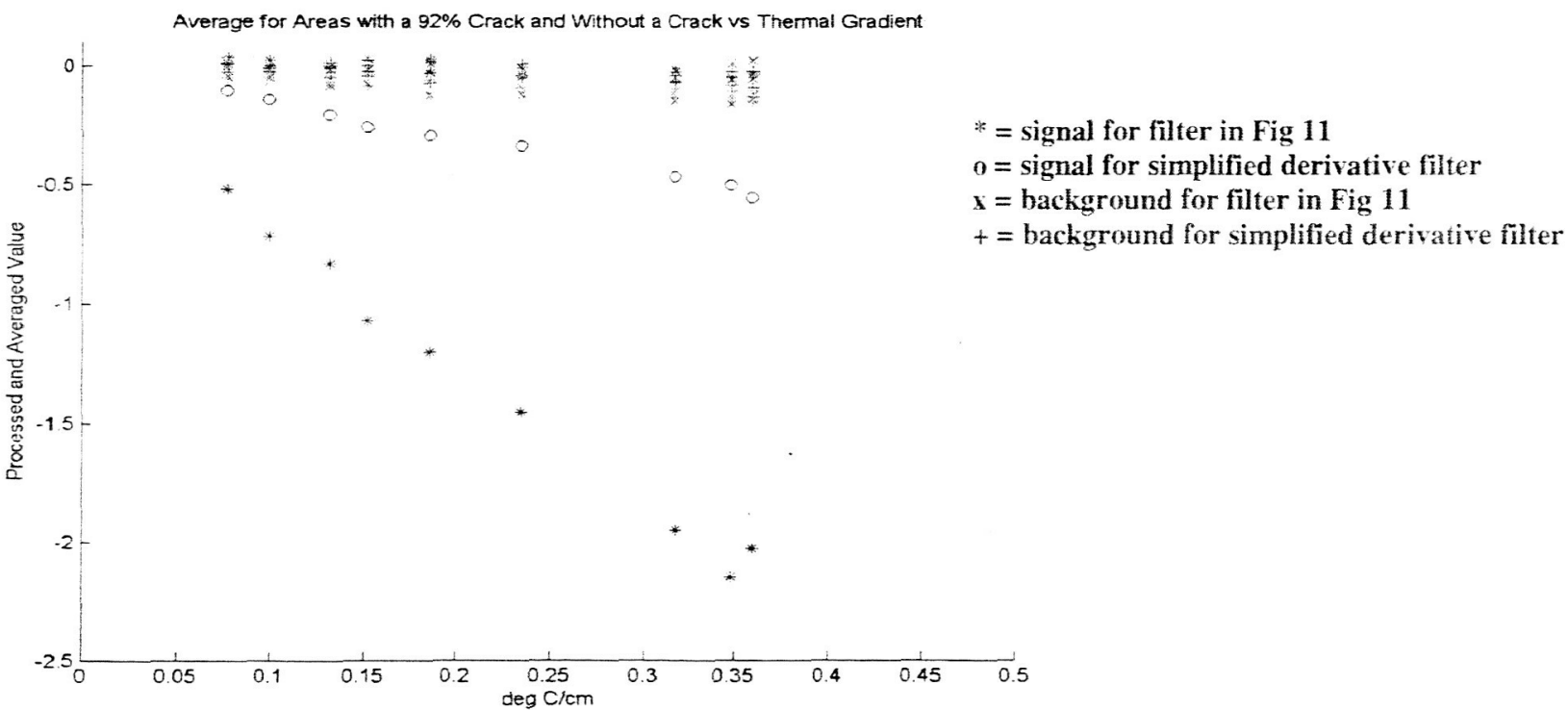

Figure 15: Signal and background plotted together 


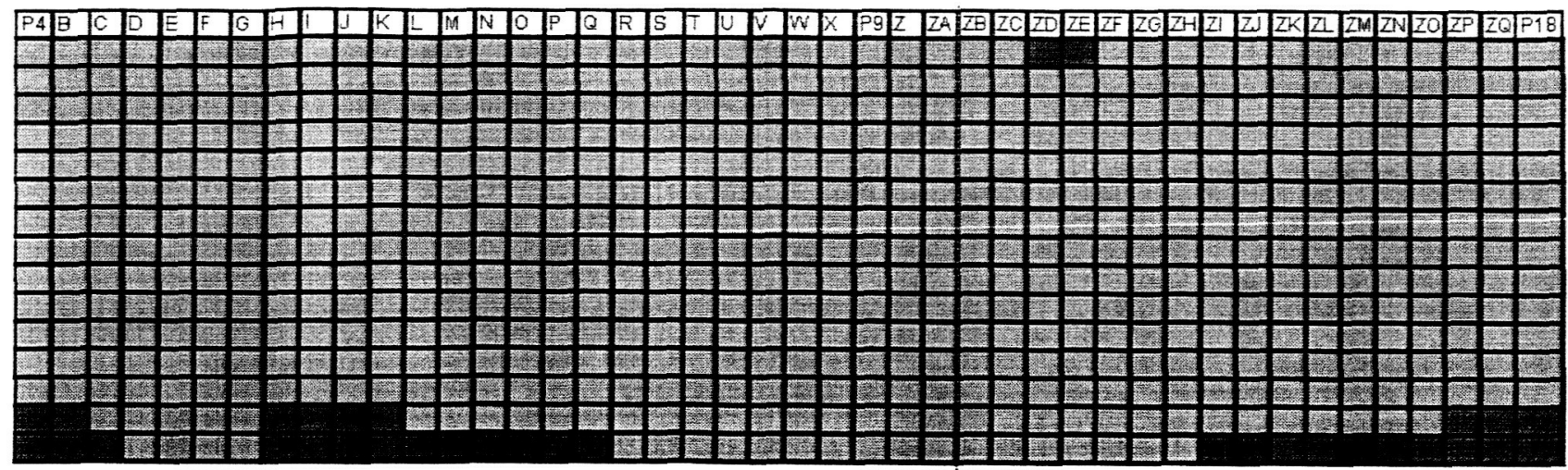

Figure 16: Map of sufficiency for $0.1^{\circ} \mathrm{C} / \mathrm{cm}$

\section{BENEFITS OF DIRECT INSPECTION BY IR (this is another candidate for cutting if we're short on space)}

IR measurements offer other advantages as well. First, since its primary goal is to measure emission, a source of illumination is not necessary. This is especially useful in someplace like low earth orbit, where illumination is plentiful for $\sim 45$ minutes a day and scarce for the other $\sim 45$, so one can easily see its potential.

It also operates at a unique portion of the electromagnetic spectrum where reflectivity or emissivity coefficients may have a higher contrast. It appears that this is the case for the SiC coating and Carbon substrate.

\section{CONCLUSION AND ACKNOWLEDGEMENTS}

All of these reasons lead one to believe that IR inspection of the Space Shuttle is extremely important to verify the integrity of its TPS and one of the optimum utilities for guaranteeing the safety of future flights.

The authors would like to also extend our deepest gratitude to the entire ATTIWG and on-orbit IR thermography development team for their professionalism and cooperation. An attempt to list them all would almost certainly lead to an oversight, however, please accept our individual thank you for helping make this paper possible.

\section{REFERENCES}

1. Gehman, H. et al. Columbia Accident Investigation Board Report Volume 1. pages 11-13, U.S. Government Printing Office, Washington, 2003.

2. O'Keefe, Sean. "Statement of Sean O'Keefe, Administrator, National Aeronautics and Space Administration; Before the Committee on Science, House of Representatives." NASA Facts. Page 1, 2003.

http://www.nasa.gov/pdf/50096main okeefe testimonv 091003.pdf

3. Gehman, H. et al. Columbia Accident Investigation Board Report Volume 1. page 174, U.S. Government Printing Office, Washington, 2003.

4. Wakata, Koichi. Development of Orbiter Thermal Protection System On-orbit Inspection and Repair. Workshop on On-orbit Servicing of Space Infrastructure Elements via Automation \& Robotics Technologies at the On-Orbit Servicing. Vancouver, Canada. 1-2 Oct, 2004. 
5. Gehman, H. et al. Columbia Accident Investigation Board Report Volume 1. pages 80-81, U.S. Government Printing Office, Washington, 2003.

6. Gehman, H. et al. Columbia Accident Investigation Board Report Volume 1. page 83, U.S. Government Printing Office, Washington, 2003.

\section{APPENDIX A}

Note: These specifications have not been verified.

\begin{tabular}{|l|l|l|l|l|}
\hline Imager & Array Size & Wavelength & Sensitivity & Technology \\
\hline QWIP (prototype) & $256 \times 256$ & $8.3 \mu \mathrm{m}$ & Uncalibrated & QWIP $(\mathrm{GaAs})$ \\
\hline LEISA (prototype) & $256 \times 256$ & $1-2.5 \mu \mathrm{m}$ & Uncalibrated & HgCdTe \\
\hline FLIR & $320 \times 240$ & LWIR & & Microbolometer \\
\hline Indigo Merlin Mid & $320 \times 256$ & $3-5 \mu \mathrm{m}$ & $<0.025 \mathrm{~K}$ & InSb \\
\hline CMC TVS-8500 & $256 \times 256$ & $3.4-4.1 \& 4.1-5.1 \mu \mathrm{m}$ & $<0.02 \mathrm{~K}\left(\right.$ at $\left.30^{\circ} \mathrm{C}\right)$ & InSb \\
\hline
\end{tabular}

